\title{
Can We Improve the Preprocessing of Photospheric Vector Magnetograms by the Inclusion of Chromospheric Observations?
}

\author{
T. Wiegelmann · J.K. Thalmann • C.J. Schrijver • \\ M.L. DeRosa • T.R. Metcalf
}

Received: 27 September 2007 / Accepted: 14 January 2008 / Published online: 2 February 2008

(C) Springer Science+Business Media B.V. 2008

\begin{abstract}
The solar magnetic field is key to understanding the physical processes in the solar atmosphere. Nonlinear force-free codes have been shown to be useful in extrapolating the coronal field upward from underlying vector boundary data. However, we can only measure the magnetic field vector routinely with high accuracy in the photosphere, and unfortunately these data do not fulfill the force-free condition. We must therefore apply some transformations to these data before nonlinear force-free extrapolation codes can be selfconsistently applied. To this end, we have developed a minimization procedure that yields a more chromosphere-like field, using the measured photospheric field vectors as input. The procedure includes force-free consistency integrals, spatial smoothing, and - newly included in the version presented here - an improved match to the field direction as inferred from fibrils as can be observed in, for example, chromospheric $\mathrm{H} \alpha$ images. We test the procedure using a model active-region field that included buoyancy forces at the photospheric level.
\end{abstract}

Unfortunately our colleague, co-author, and friend Tom Metcalf passed away before the final manuscript was finished. We continued our joint work in his memory and would like to remember him here.

T. Wiegelmann $(\bowtie) \cdot$ J.K. Thalmann

Max-Planck-Institut für Sonnensystemforschung, Max-Planck-Strasse 2, 37191 Katlenburg-Lindau, Germany

e-mail: wiegelmann@mps.mpg.de

J.K. Thalmann

e-mail: thalmann@mps.mpg.de

C.J. Schrijver · M.L. DeRosa

Department ADBS, Lockheed Martin Advanced Technology Center, Building 252, 3251 Hanover Street, Palo Alto, CA 94304, USA

C.J. Schrijver

e-mail: schryver@Imsal.com

M.L. DeRosa

e-mail: derosa@Imsal.com

T.R. Metcalf

Colorado Research Associates Division, Northwest Research Associates, 3380 Mitchell Lane, Boulder, CO 90301, USA 
The proposed preprocessing method allows us to approximate the chromospheric vector field to within a few degrees and the free energy in the coronal field to within one percent.

Keywords Magnetic fields · Photosphere $\cdot$ Chromosphere · Corona

\section{Introduction}

The solar interior, photosphere, and atmosphere are coupled by magnetic fields. It is therefore important to gain insights about the magnetic field structure in all layers of the Sun and solar atmosphere. Direct and accurate measurements of the magnetic field vector are typically carried out only on the photosphere. Although measurements in higher layers are available for a few individual cases, e.g., in the chromosphere by Solanki et al. (2003) and in the corona by Lin, Kuhn, and Coulter (2004), the line-of-sight integrated character of such chromospheric and coronal magnetic field measurements complicates their interpretation (Kramar, Inhester, and Solanki, 2006). Knowledge of the magnetic field in the corona is essential, however, to understand basic physical processes such as the onset of flares, coronal mass ejections, and eruptive prominences.

Inferences of the coronal magnetic field can be obtained by extrapolating measurements of the photospheric magnetic field vector (e.g., observed by Hinode/SOT, SOLIS, or the upcoming SDO/HMI instruments) into the corona. Because the magnetic pressure dominates the plasma pressure in active-region coronae, making the plasma $\beta$ low (see work by Gary (2001), and Schrijver and van Ballegooijen (2005), which discuss the plasma $\beta$ over active regions and over the quiet Sun, respectively), these extrapolations neglect nonmagnetic forces and assume the coronal magnetic field $\mathbf{B}$ to be force-free, such that it obeys

$$
\begin{aligned}
\nabla \cdot \mathbf{B} & =0, \\
(\nabla \times \mathbf{B}) \times \mathbf{B} & =0 .
\end{aligned}
$$

Equation (2) implies that the electric current density $\mu_{0} \mathbf{j}=\nabla \times \mathbf{B}$ is parallel to the magnetic field B. Starting more than a quarter century ago (Sakurai, 1981), different mathematical methods and numerical implementations have been developed to solve the nonlinear force-free equations (1) and (2) for the solar case. See, for example, Sakurai (1989), Amari et al. (1997), and Wiegelmann (2008) for review papers and Schrijver et al. (2006) and Metcalf et al. (2007) for evaluations of the performance of corresponding computer programs with model data. The codes use the magnetic field vector (or quantities derived from the magnetic field vector) on the bottom boundary of a computational domain as input. One would like to prescribe the measured photospheric data as the bottom boundary of nonlinear force-free fields (NLFFF) codes, but there is a problem: The observed photospheric magnetic field is usually not force-free. The relatively high plasma $\beta$ in the photosphere means that nonmagnetic forces cannot be neglected there and that such photospheric magnetic field data are not consistent with well-known force-free compatibility conditions defined in Aly (1989). Recently, Wiegelmann, Inhester, and Sakurai (2006) developed a scheme that mitigates this problem, in which the inconsistent and noisy photospheric vector magnetograms used as bottom boundary conditions are preprocessed to remove net magnetic forces and torques and to smooth out small-scale noise-like magnetic structures. The resulting magnetic field data are sufficiently force-free and smooth for use with extrapolation codes, but they also are found to bear a high resemblance to chromospheric vector magnetic field data. This leads us to the question of whether we can constrain the preprocessing tool further by taking direct chromospheric observations, such as $\mathrm{H} \alpha$ images, into consideration. We will investigate this topic in the present work. 


\section{A Short Review of Consistency Criteria for Force-Free Coronal Extrapolations}

In this section, we briefly discuss the criteria on the photospheric boundary data that are required for consistency with a force-free extrapolation of the overlying coronal magnetic field. Molodensky (1969), Molodensky (1974), Aly (1989), and Sakurai (1989) show how moments of the Lorentz force, integrated over a volume of interest, define constraints on the closed surface bounding this volume. As explained in detail in Molodensky (1974) the sense of these relations is that on average a force-free field cannot exert pressure on the boundary or shear stresses along axes lying in the boundary. For the coronal magnetic field extrapolation calculations discussed here, a localized region of interest, such as an active region, is typically selected for analysis. The extrapolation algorithms applied to the coronal volume overlying such localized regions of interest require boundary conditions, and, except at the lower (photospheric) boundary, these boundary conditions are usually chosen to be consistent with potential fields and thus do not possess magnetic forces or torques. In these cases, the consistency criteria reduce to conditions on the lower boundary only:

1. On average, force-free fields cannot exert pressure on the boundary:

$$
\begin{aligned}
& F_{1}=\int_{S} B_{x} B_{z} \mathrm{~d} x \mathrm{~d} y=0, \\
& F_{2}=\int_{S} B_{y} B_{z} \mathrm{~d} x \mathrm{~d} y=0, \\
& F_{3}=\int_{S}\left(B_{x}^{2}+B_{y}^{2}\right) \mathrm{d} x \mathrm{~d} y-\int_{S} B_{z}^{2} \mathrm{~d} x \mathrm{~d} y=0 .
\end{aligned}
$$

2. On average, force-free fields cannot create shear stresses along axes lying in the boundary:

$$
\begin{aligned}
& T_{1}=\int_{S} x\left(B_{x}^{2}+B_{y}^{2}\right) \mathrm{d} x \mathrm{~d} y-\int_{S} x B_{z}^{2} \mathrm{~d} x \mathrm{~d} y=0, \\
& T_{2}=\int_{S} y\left(B_{x}^{2}+B_{y}^{2}\right) \mathrm{d} x \mathrm{~d} y-\int_{S} y B_{z}^{2} \mathrm{~d} x \mathrm{~d} y=0, \\
& T_{3}=\int_{S} y B_{x} B_{z} \mathrm{~d} x \mathrm{~d} y-\int_{S} x B_{y} B_{z} \mathrm{~d} x \mathrm{~d} y=0 .
\end{aligned}
$$

These relations must be fulfilled to be suitable boundary conditions for a nonlinear force-free coronal magnetic field extrapolation. We define the dimensionless numbers

$$
\begin{aligned}
\epsilon_{\text {force }} & =\frac{\left|F_{1}\right|+\left|F_{2}\right|+\left|F_{3}\right|}{\int_{S}\left(B_{x}^{2}+B_{y}^{2}+B_{z}^{2}\right) \mathrm{d} x \mathrm{~d} y}, \\
\epsilon_{\text {torque }} & =\frac{\left|T_{1}\right|+\left|T_{2}\right|+\left|T_{3}\right|}{\int_{S} \sqrt{x^{2}+y^{2}}\left(B_{x}^{2}+B_{y}^{2}+B_{z}^{2}\right) \mathrm{d} x \mathrm{~d} y}
\end{aligned}
$$

to evaluate how well these criteria are met. Ideally, it is necessary for $\epsilon_{\text {force }}=\epsilon_{\text {torque }}=0$ for a force-free coronal magnetic field to exist. 
Aly (1989) pointed out that the magnetic field is probably not force-free in the photosphere where $\mathbf{B}$ is measured, because the plasma $\beta$ in the photosphere is of the order of unity and pressure gradient and gravity forces are not negligible. The integral relations (3) $-(8)$ are not satisfied in this case in the photosphere and the measured photospheric field is not a suitable boundary condition for a force-free extrapolation. Investigations by Metcalf et al. (1995) revealed that the solar magnetic field is not force-free in the photosphere but becomes force-free about $400 \mathrm{~km}$ above the photosphere. The problem has been addressed also by Gary (2001), who pointed out that care has to be taken when extrapolating the coronal magnetic field as a force-free field from photospheric measurements, because the force-free low corona is sandwiched between two regions (photosphere and higher corona) with a plasma $\beta \approx 1$, where the force-free assumption might break down. An additional problem is that measurements of the photospheric magnetic vector field contain inconsistencies and noise. In particular the components of $\mathbf{B}$ transverse to the line of sight, as measured by current vector magnetographs, are more uncertain than the line-of-sight component. Because measurements in higher layers of the solar atmosphere (where the magnetic field is force-free) are not routinely available, we have to deal with the problem of inconsistent (with the forcefree assumption as defined by Equations (3) - (8)) photospheric measurements. A routine that uses measured photospheric vector magnetograms to find suitable boundary conditions for a nonlinear force-free coronal magnetic field extrapolation, dubbed "preprocessing," has been developed by Wiegelmann, Inhester, and Sakurai (2006).

\section{Preprocessing}

\subsection{Classical Preprocessing}

The preprocessing scheme of Wiegelmann, Inhester, and Sakurai (2006) involves minimizing a two-dimensional functional of quadratic form similar to the following:

$$
L_{\text {prep }}=\mu_{1} L_{1}+\mu_{2} L_{2}+\mu_{3} L_{3}+\mu_{4} L_{4}+\mu_{5} L_{5},
$$

where

$$
\begin{aligned}
L_{1}= & {\left[\left(\sum_{p} B_{x} B_{z}\right)^{2}+\left(\sum_{p} B_{y} B_{z}\right)^{2}+\left(\sum_{p} B_{z}^{2}-B_{x}^{2}-B_{y}^{2}\right)^{2}\right], } \\
L_{2}= & {\left[\left(\sum_{p} x\left(B_{z}^{2}-B_{x}^{2}-B_{y}^{2}\right)\right)^{2}+\left(\sum_{p} y\left(B_{z}^{2}-B_{x}^{2}-B_{y}^{2}\right)\right)^{2}\right.} \\
& \left.+\left(\sum_{p} y B_{x} B_{z}-x B_{y} B_{z}\right)^{2}\right], \\
L_{3}= & {\left[\sum_{p}\left(B_{x}-B_{x \mathrm{obs}}\right)^{2}+\sum_{p}\left(B_{y}-B_{y \mathrm{obs}}\right)^{2}\right.} \\
& \left.+\sum_{p}\left(B_{z}-B_{z \mathrm{obs}}\right)^{2}\right], \\
L_{4}= & {\left[\sum_{p}\left(\Delta B_{x}\right)^{2}+\left(\Delta B_{y}\right)^{2}+\left(\Delta B_{z}\right)^{2}\right] . }
\end{aligned}
$$


The surface integrals as defined in Equations (3) - (8) are here replaced by a summation $\sum_{p}$ over all grid nodes $p$ of the bottom surface grid. We normalize the magnetic field strength with the average magnetic field on the photosphere and the length scale with the size of the magnetogram. Each constraint $L_{n}$ is weighted by a yet undetermined factor $\mu_{n}$. The first term $(n=1)$ corresponds to the force-balance conditions (3) - (5) and the next $(n=2)$ to the torque-free condition (6) - (8). The following term $(n=3)$ contains the difference of the optimized boundary condition with the measured photospheric data and the next term $(n=4)$ controls the smoothing. The 2D Laplace operator is designated by $\Delta$ and the differentiation in the smoothing term is achieved by the usual five-point stencil. The last term $(n=5)$ has not been used in preprocessing so far and will be introduced in the next section. The aim of the preprocessing procedure is to minimize $L_{\text {prep }}$ so that all terms $L_{n}$ if possible are made small simultaneously. This minimization procedure provides us iterative equations for $B_{x}, B_{y}, B_{z}$ (see Wiegelmann, Inhester, and Sakurai (2006) for details). As a result of the preprocessing we get a data set that is consistent with the assumption of a force-free magnetic field in the corona but also as close as possible to the measured data within the noise level.

Nonlinear force-free extrapolation codes can be applied only to low plasma $\beta$ regions, where the force-free assumption is justified. This is known not to be the case in the photosphere, but it is mostly true for the upper chromosphere and for the corona in quiescent conditions. The preprocessing scheme as used until now modifies observed photospheric vector magnetograms with the aim of approximating the magnetic field vector at the bottom of the force-free domain (i.e., at a height that we assume to be located in the middle to upper chromosphere). In this study, we investigate whether the use of chromospheric fibril observations as an additional constraint in the preprocessing can bring the resulting field into even better agreement with the expected chromospheric vector field.

We discuss this idea in the next section.

\section{2. $\mathrm{H} \alpha$ Preprocessing}

The idea is to specify another term $\left(\mu_{5} L_{5}\right)$ in Equation (11) that measures how well the preprocessed magnetic field is aligned with fibrils seen in $\mathrm{H} \alpha$. As a first step we have to extract the directions of the fibrils, say $H_{x}$ and $H_{y}$, out of the Ho images, where $\mathbf{H}$ is a unit vector tangent $(|\mathbf{H}|=1)$ to the chromospheric fibrils projected onto the solar photosphere (representing the field direction with a $180^{\circ}$ ambiguity). For simplicity one might rebin $H_{x}$ and $H_{y}$ to the same resolution as the vector magnetogram. In regions where we cannot identify clear filamentary structures in the images we set $H_{x}=H_{y}=0$. These regions are only affected by the other, classical terms of the preprocessing functional (11). The angle of the projected magnetic field vector on the $x y$-plane with the $\mathrm{H} \alpha$ image is

$$
\sin (\phi)=\frac{\left|\mathbf{B}_{\|} \times \mathbf{H}\right|}{\left|\mathbf{B}_{\|}\right||\mathbf{H}|},
$$

where $\mathbf{B}_{\|}=\left(B_{x}, B_{y}\right)$ is the projection of the magnetic field vector in the $x y$-plane and $\mathbf{H}=\left(H_{x}, H_{y}\right)$ is the direction of the chromospheric Ho fibrils. The preprocessing aims at deriving the magnetic field vector on the bottom boundary of the force-free domain, which is located in the chromosphere. The chromospheric magnetic field is certainly a priori unknown and as initial condition for the preprocessing routine we take $\mathbf{B}_{\|}$from the photospheric vector magnetogram. 
We define the functional

$$
L_{5}=\sum_{p} w\left(B_{x} H_{y}-B_{y} H_{x}\right)^{2}=\sum_{p} w \mathbf{B}_{\|}^{2} \sin ^{2}(\phi) .
$$

Note that the term $B_{x} H_{y}-B_{y} H_{x}$ in Equation (17) weights the angle with the magnetic field strength, because it is in particularly important in minimizing the angle in strong-field regions. The space-dependent function $w=w(x, y)$ is not a priori related to the magnetic field strength and can be specified to indicate the confidence level of the fibril directionfinding algorithm (see, e.g., Inhester, Feng, and Wiegelmann (2007) for the description of a corresponding feature recognition tool). For the application to observational data, $w$ will be (with appropriate normalization) provided by this tool. It is likely, however, that the direction of the $\mathrm{H} \alpha$ fibrils can be identified more accurately in strong magnetic field regions, but this is not an a priori assumption. In Section 4.3 we investigate the influence of different assumptions for $w$.

We take the functional derivative of $L_{5}$ :

$$
\frac{\mathrm{d} L_{5}}{\mathrm{~d} t}=2\left(B_{x} H_{y}-B_{y} H_{x}\right)\left(H_{y} \frac{\mathrm{d} B_{x}}{\mathrm{~d} t}-H_{x} \frac{\mathrm{d} B_{y}}{\mathrm{~d} t}\right) .
$$

For a sufficiently small time step $d t$ we get a decreasing $L_{5}$ with the iteration equations

$$
\begin{aligned}
& \frac{\mathrm{d} B_{x}}{\mathrm{~d} t}=-2 w \mu_{5}\left(B_{x} H_{y}-B_{y} H_{x}\right) H_{y}, \\
& \frac{\mathrm{d} B_{y}}{\mathrm{~d} t}=2 w \mu_{5}\left(B_{x} H_{y}-B_{y} H_{x}\right) H_{x} .
\end{aligned}
$$

The aim of our procedure is to make all terms in functional (11) small simultaneously. There are obvious contradictions between some of the $L_{n}$ terms, such as between the $n=3$ (photospheric data) and $n=4$ (smoothing) terms. An important task is to find suitable values for the five parameters $\mu_{n}$ that control the relative weighting of the terms in Equation (11). The absolute values do not matter; only the relative weightings are important. We typically give all integral relations of the force and torque conditions (3) - (8) the same weighting (unity). To fulfill these consistency integrals is essential for finding suitable boundary conditions for a nonlinear force-free extrapolation. In principle it would be possible to examine different values for the force-free term $\mu_{1}$ and torque-free term $\mu_{2}-$ or even to give six different weightings for the six integral relations - but giving all integrals the same weighting seems to be a reasonable choice. The torque integrals depend on the choice of the length scale $D$ and giving the same weighting to all integrals requires $\mu_{2}=\frac{\mu_{1}}{D^{2}}$. For the length-scale normalization used here $(D=1)$ this leads to $\mu_{1}=\mu_{2}$.

We will test our newly developed method with the help of a model active region in the next section.

\section{Tests}

\subsection{An Active Region Model for Testing the New Method}

We test our extended preprocessing routine with the help of an active-region model recently developed by van Ballegooijen et al. (2007). In this model line-of-sight photospheric measurements from SOHO/MDI have been used to compute a potential field. A twisted flux 


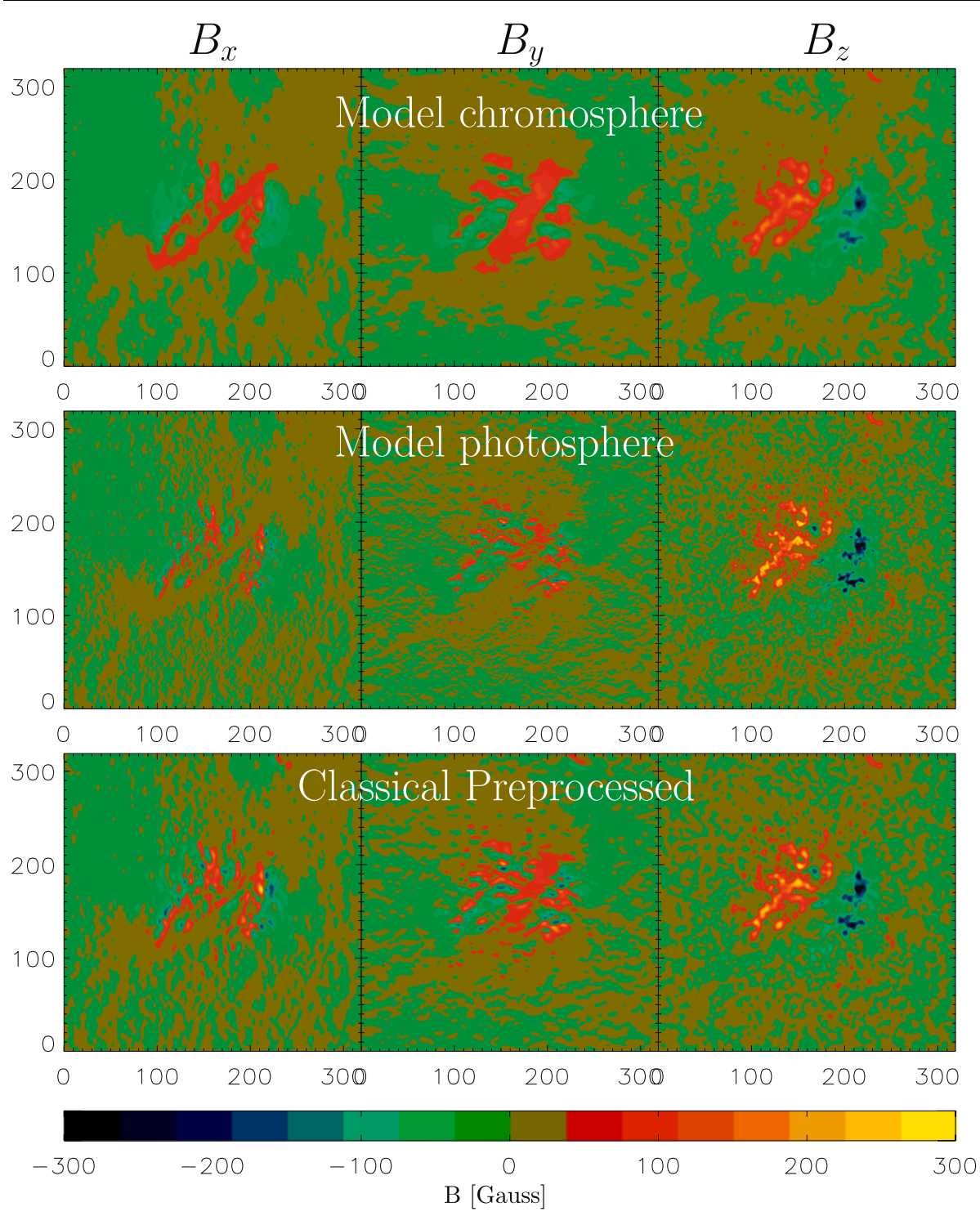

Figure 1 Top: Model chromospheric magnetic field located in the $z=2$ layer. Center: Model photospheric magnetic field. Bottom: Model photospheric magnetic field after classical preprocessing with $\mu_{3}=0.025$, $\mu_{4}=0.155$.

rope was then inserted into the volume, after which the whole system was relaxed toward a nonlinear force-free state with the magnetofrictional method described in van Ballegooijen (2004). The van Ballegooijen et al. (2007) model is force-free throughout the entire computational domain, except within two gridpoints of the bottom boundary. Hereafter, we refer to the bottom of the force-free layer as the "model chromosphere" (see the top panel of Figure 1). On the bottom boundary (see the central panel of Figure 1), hereafter referred to as the "model photosphere," the model contains significant nonmagnetic forces and the 
force-free consistency criteria (3) - (8) are not satisfied. These forces take the form of vertical buoyancy forces directed upward and have been introduced by van Ballegooijen et al. (2007) to mimic the effect of a reduced gas pressure in photospheric flux tubes. The nature of these forces is therefore expected to be similar to those observed on the real Sun. For a more detailed discussion we refer to Metcalf et al. (2007) Both the chromospheric $\left(\mathbf{B}_{\mathrm{ch}}\right)$ as well as the photospheric magnetic field vector $\left(\mathbf{B}_{\mathrm{ph}}\right)$ from the van Ballegooijen et al. (2007) model have been used to test four sophisticated nonlinear force-free extrapolation codes in a blind algorithm test by Metcalf et al. (2007). ${ }^{1}$ The codes computed nonlinear force-free codes in a $320 \times 320 \times 256$ box, which is about at the upper limit current codes can handle on workstations. We briefly summarize the results of Metcalf et al. (2007) as follows:

- NLFFF extrapolations from model chromospheric data recover the original reference field with high accuracy.

- When the extrapolations are applied to the model photospheric data, the reference field is not well recovered.

- Preprocessing of the model photospheric data to remove net forces and torques improves the result, but the resulting accuracy was lower than for extrapolations from the model chromospheric data.

The poor performance of extrapolations using the unprocessed model photospheric data is related to their inconsistency with respect to the force-free conditions (3) - (8). The central panel of Figure 1 shows the photospheric magnetic field and the central panel of Figure 2 illustrates the difference between the model chromospheric and model photospheric fields. It is evident that there are remarkable differences in all components of the magnetic field vector. For real data we usually cannot measure the chromospheric magnetic field vector directly (which was possible for van Ballegooijen et al. (2007) model data) and we have to apply preprocessing before using the data as input for force-free extrapolation codes. Force-free extrapolations using preprocessed data from the model photosphere (as lower panels of Figures 1 and 2), although encouraging, were not completely satisfactory, in light of the results being worse than when the model chromospheric data were used as boundary conditions. In what follows, we will use an artificial $\mathrm{H} \alpha$ image created from the model chromosphere to test a modified preprocessing scheme and compare the results to the classical (original) preprocessing scheme.

We use the model chromospheric magnetic field $\left(\mathbf{B}_{\mathrm{ch}}\right)$ to derive the direction vectors of the artificial $\mathrm{H} \alpha$ images. For the model case we can simply use the chromospheric model field to specify the direction vectors $H_{x}$ and $H_{y}$, which contain only information regarding the direction of the horizontal components of the magnetic field (including a $180^{\circ}$ ambiguity) but no information about the magnetic field strength. For real data this information can be derived from high-resolution $\mathrm{H} \alpha$ images by using feature recognition techniques (e.g., the ridge detector of Inhester, Feng, and Wiegelmann (2007)).

\subsection{Optimal Parameter Set for Classical Preprocessing}

We tested more than 1000 possible combinations of $\mu_{3}$ and $\mu_{4}$ using the model-photospheric field as input and computed the Pearson correlation coefficient between the preprocessed results and the model chromospheric field. Only $B_{x}$ and $B_{y}$ were used in computing the correlation coefficient, because the correlation of the longitudinal (i.e., the line-of-sight)

\footnotetext{
${ }^{1}$ Previously, the NLFFF codes have been intensively tested and evaluated with the Low and Lou (1990) semi-analytic equilibria (Schrijver et al., 2006).
} 




Figure 2 Top: Model chromospheric magnetic field. Center: Difference between the chromospheric and photospheric model vector fields. Bottom: Difference between the chromospheric and classical preprocessed photospheric fields.

component is in general higher than that of the transverse components, because $B_{z}$ is not being affected by the ambiguity problem and the noise is much lower than in the other directions.

We computed 100 combinations of $\mu_{3}$ and $\mu_{4}$ for $-0.2 \leq \mu_{3}$ and $\mu_{4} \leq 0.2$ with a step size of $\Delta \mu_{3}=\Delta \mu_{4}=0.05$. Hereafter a local maximum around $\mu_{3}=0.05$ and $\mu_{4}=0.15$ appeared. This region was analyzed in more detail by using these two values as new initial guess. To do this, we tried another 100 combinations around this pair with a reduced step 

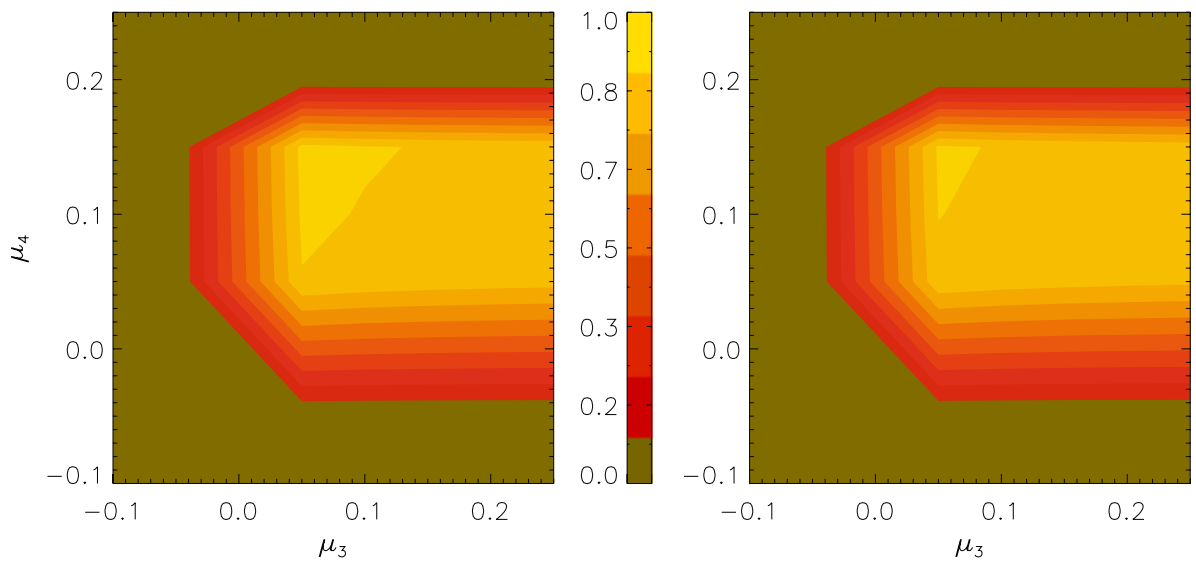

Figure 3 Correlation of the preprocessed field (left panel: $B_{x}$, right panel: $B_{y}$ ) with the model chromosphere depending on the preprocessing parameters $\mu_{3}$ and $\mu_{4}$. We found a maximum correlation at $\mu_{3}=0.025$ and $\mu_{4}=0.155$.

size of $\Delta \mu_{3}=\Delta \mu_{4}=0.005$ in the positive as well as the negative direction. Then the absolute maximum of the correlation coefficients for both $B_{x}$ and $B_{y}$ appeared at $\mu_{3}=$ 0.025 and $\mu_{4}=0.155$ (see Figure 3). The bottom panel of Figure 1 shows the corresponding preprocessed photospheric magnetic field.

\subsection{Optimal Parameters and Weighting Functions for $\mathrm{H} \alpha$ Preprocessing}

In the following we aim to find suitable parameters for including information from $\mathrm{H} \alpha$ images into the preprocessing.

Our main goal is to investigate the effects of additional chromospheric information. To exclude side effects we therefore keep the combination $\mu_{1}-\mu_{4}$ combination found in the previous section to be able to clearly investigate the effect of the additional term $L_{5}$. In principle one could vary all $\mu_{n}$ simultaneously. We cannot exclude that there might exist a better combination of $\mu_{1}$ to $\mu_{5}$ with better agreement of our preprocessed field and the model chromospheric field. This is, however, not the aim of this work, because this is not a suitable way to deal with real data, because there is no model chromosphere to test the result. It is not possible to provide an optimal parameter set suitable for all vector magnetographs. The optimal combination has to be carried out for different instruments separately. We expect that an optimal parameter set for a certain instrument and particular region will also be useful for the preprocessing of other regions of the same kind (say active regions) observed with the same instrument.

We test our methods with "model fibrils" extracted from the model chromosphere shown in the top panel of Figure 4. We define $w(x, y)$ used in Equation (17) as one of the following:

1. We assume that every point of our $\mathrm{H} \alpha$ image gives us the exact orientation of the magnetic field (which is indeed the case, as we calculated it from the chromospheric model data) and fix our weighting with $w(x, y)=w_{1}=1$.

2. We assume that the photospheric magnetic field magnitude gives us the importance of the $\mathrm{H} \alpha$ information at each point and use

$$
w(x, y)=w_{2}=\sqrt{\left(B_{x}^{2}+B_{y}^{2}+B_{z}^{2}\right)_{\mathrm{ph}}} .
$$




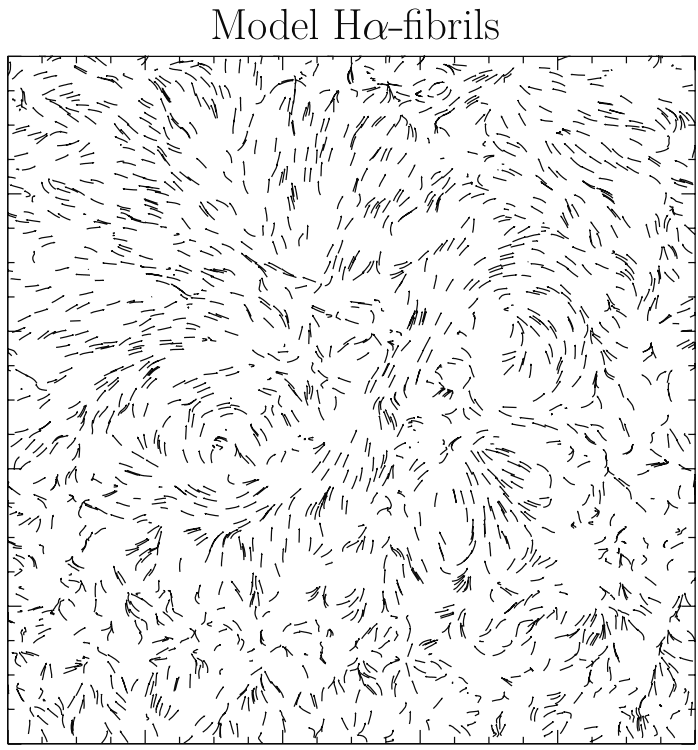

$w_{2}$

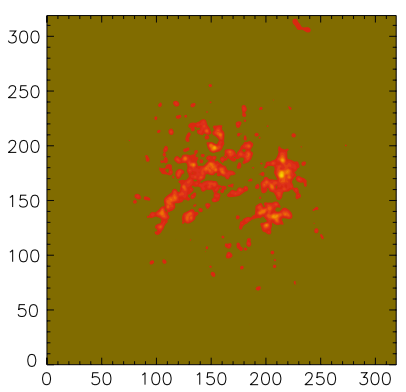

0.0 $w_{3}$

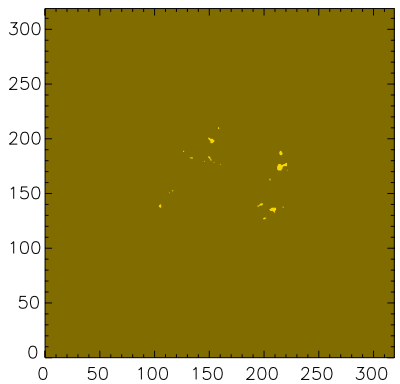

$w_{4}$

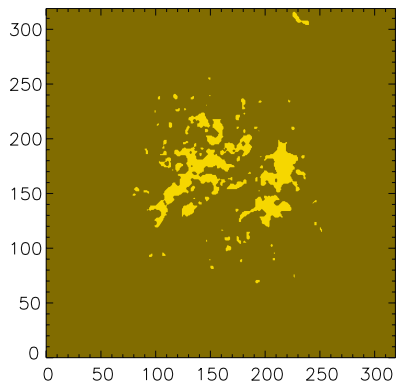

Figure 4 Top: $\mathrm{H} \alpha$ fibrils identified from the model chromosphere. The fibrils give us information about the transverse components $\left(B_{x}\right.$ and $\left.B_{y}\right)$ of the chromospheric magnetic field. The fibrils contain a $180^{\circ}$ ambiguity and do not provide any information about the chromospheric magnetic field strength. Bottom: Different weighting functions $w_{2}, w_{3}$, and $w_{4}$ (from left to right, respectively). Regions where $w$ is higher are more important in the $L_{5}$ preprocessing term (17), which controls the influence of the H $\alpha$ fibrils.

We scale $w_{2}$ to a maximum value of 1 . (See Figure 4, bottom left panel.)

3. We do as in the previous case but assume now that only points in the magnetogram where the field magnitude is greater than $50 \%$ of the maximum contribute to the $\mathrm{H} \alpha$ preprocessing. So, we define

$$
w(x, y)=w_{3}= \begin{cases}1 & \text { for } w_{2} \geq 0.5 \\ 0 & \text { for } w_{2}<0.5\end{cases}
$$

(See Figure 4, bottom center panel.) 
Table 1 Results of the various preprocessing schemes: the model chromosphere and photosphere (first two rows), classical preprocessing (third row), and the $\mathrm{H} \alpha$ preprocessing cases (last four rows). Column 1 identifies the data set and columns 2 and 3 the value of $\mu_{5}$ and the weighting scheme used for the H $\alpha$ preprocessing cases. Columns $4-7$ provide the value of the functionals $L_{12}=L_{1}+L_{2}, L_{3}, L_{4}$, and $L_{5}$ as defined in Equations (12) - (15) and (17), respectively. In columns 8 and 9 we show how well the force-free and torque-free consistency criteria ( $\left.\varepsilon_{\text {force }}, \varepsilon_{\text {torque }}\right)$ as defined in Equations (9) and (10) are fulfilled. The last two columns contain the averaged angle $\left[\phi_{\text {ave }}=\langle\phi(x, y)\rangle\right]$ of the field with the model chromospheric data and a magneticfield-weighted average angle $\left[\phi_{\mathrm{ave}, \mathrm{w}}=\left\langle B^{2} \phi(x, y)\right\rangle /\left\langle B^{2}\right\rangle\right]$ with $\phi(x, y)$ as defined in Equation (16).

\begin{tabular}{|c|c|c|c|c|c|c|c|c|c|c|}
\hline \multirow[t]{2}{*}{ Data } & \multicolumn{2}{|c|}{ Weights } & \multicolumn{4}{|c|}{$L_{\text {prep }} \times 10^{-6}$} & \multicolumn{2}{|c|}{ Aly criteria } & \multirow{2}{*}{$\begin{array}{l}\phi_{\text {ave }} \\
(\mathrm{deg})\end{array}$} & \multirow{2}{*}{$\begin{array}{l}\phi_{\text {ave }, w} \\
\text { (deg) }\end{array}$} \\
\hline & $\overline{\mu_{5}}$ & $w$ & $L_{12}$ & $L_{3}$ & $L_{4}$ & $L_{5}$ & $\varepsilon_{\text {force }}$ & $\varepsilon_{\text {torque }}$ & & \\
\hline$B_{\mathrm{ch}}$ & - & - & 452.137 & 3.57 & 0.18 & 0.00 & 0.0171 & 0.0203 & - & - \\
\hline$B_{\mathrm{np}}$ & - & - & 338287 & 0.00 & 4.45 & 0.49 & 0.4138 & 0.5797 & 19.2 & 18.9 \\
\hline$B_{\mathrm{cp}}$ & - & - & 0.06658 & 2.30 & 0.18 & 0.21 & 0.0003 & 0.0001 & 10.1 & 8.8 \\
\hline$B_{\mathrm{H \alpha p}}$ & 1.525 & $w_{1}$ & 33.37 & 2.45 & 0.17 & 0.0007 & 0.0062 & 0.0011 & 1.1 & 0.4 \\
\hline$B_{\mathrm{H \alpha p}}$ & 1.765 & $w_{2}$ & 31.70 & 2.47 & 0.15 & 0.0171 & 0.0060 & 0.0012 & 7.3 & 2.0 \\
\hline$B_{\mathrm{H} \alpha \mathrm{p}}$ & 1.880 & $w_{3}$ & 29.10 & 2.41 & 0.15 & 0.1355 & 0.0058 & 0.0012 & 10.8 & 6.8 \\
\hline$B_{\mathrm{H} \alpha \mathrm{p}}$ & 2.115 & $w_{4}$ & 32.16 & 2.41 & 0.17 & 0.0531 & 0.0060 & 0.0012 & 10.4 & 3.1 \\
\hline
\end{tabular}

4. In our last case we assume in the same way as in the previous one, but now only points in the magnetogram where the field magnitude is greater than $10 \%$ of the maximum contribute to the preprocessing. All these grid points are weighted with 1 and the rest with zero. In other words, one defines

$$
w(x, y)=w_{4}= \begin{cases}1 & \text { for } w_{2} \geq 0.1 \\ 0 & \text { for } w_{2}<0.1\end{cases}
$$

(See Figure 4 bottom right panel.)

We now figure out the optimal value of $\mu_{5}$ in Equation (11) for the four different weighting functions $w_{1}-w_{4}$. Initially, we use a step size of $\Delta \mu_{5}=0.05$ and then, around the first appearing maximum, we reduced it to $\Delta \mu_{5}=0.005$. This is to find a more precise optimal value of $\mu_{5}$. We calculate the Pearson correlation coefficient between the chromospheric reference field $\left(\mathbf{B}_{\mathrm{ch}}\right)$ and the minimum solution of the preprocessing routine $\left(\mathbf{B}_{\mathrm{pp}}\right)$. This provides us the optimal values of $\mu_{5}$ for the different weighting functions (see the second column in Table 1).

\section{Results}

Table 1 lists some metrics related to the various preprocessing schemes, including the dimensionless numbers $\epsilon_{\text {force }}$ and $\epsilon_{\text {torque }}$ from Equations (9) and (10), the values of the various $L_{n}$ from Section 3, and the averaged angles between the preprocessing results and the model chromospheric field. The first three rows of the table list the model chromosphere $\left(B_{\mathrm{ch}}\right)$ and photosphere $\left(B_{\mathrm{ph}}\right)$ data and the classical preprocessing scheme $\left(B_{\mathrm{cp}}\right)$. When using the unprocessed model photospheric data $\left(B_{\mathrm{ph}}\right)$, it is clear that the force-free consistency criteria (as represented by $L_{12}, \varepsilon_{\text {force }}$, and $\varepsilon_{\text {torque }}$ ) are not fulfilled and are orders of magnitude higher than for the chromospheric data $\left(B_{\mathrm{ch}}\right)$. Consequently, we cannot expect the extrapolation codes to result in a meaningful nonlinear force-free field in the corona, as discussed in Metcalf et al. (2007). 
Table 2 Results of the nonlinear force-free field extrapolations in a $3 \mathrm{D}$ box $(320 \times 320 \times 256)$. The rows are the same as in Table 1. The first three columns identify the preprocessing scheme, the value of $\mu_{5}$, and the weighting scheme as in Table 1. Column 4 contains the functional $L$, as defined in Equation (21), which tells us how well the force-free and solenoidal conditions are fulfilled in the computational box. In columns $5-9$ we compare the extrapolated 3D magnetic field with the reference solution and use different quantitative comparison metrics: the vector correlation $\left(C_{\mathrm{vec}}\right)$, the Cauchy - Schwarz metric $\left(C_{\mathrm{cs}}\right)$, the complement of the normalized vector error $\left(E_{\mathrm{n}}^{\prime}\right)$, the complement of the mean vector error $\left(E_{\mathrm{m}}^{\prime}\right)$, and the total magnetic energy normalized to the reference field $\left(\epsilon_{\mathrm{mag}}\right)$ as defined in Equations $(22)-(26)$, respectively. Perfect agreement for any of these comparison metrics is unity.

\begin{tabular}{lllllllll}
\hline Data & $\mu_{5}$ & $w$ & $L$ & $C_{\mathrm{vec}}$ & $C_{\mathrm{cs}}$ & $E_{\mathrm{n}}^{\prime}$ & $E_{\mathrm{m}}^{\prime}$ & $\epsilon_{\mathrm{mag}}$ \\
\hline$B_{\mathrm{ch}}$ & - & - & 0.53 & 1.00 & 1.00 & 1.00 & 1.00 & 1.00 \\
$B_{\mathrm{np}}$ & - & - & 46.03 & 0.91 & 0.99 & 0.69 & 0.85 & 0.65 \\
$B_{\mathrm{cp}}$ & - & - & 5.99 & 0.97 & 0.99 & 0.80 & 0.85 & 0.97 \\
$B_{\mathrm{H} \alpha \mathrm{p}}$ & 1.525 & $w_{1}$ & 3.45 & 0.97 & 1.00 & 0.81 & 0.85 & 1.01 \\
$B_{\mathrm{H} \alpha \mathrm{p}}$ & 1.765 & $w_{2}$ & 2.37 & 0.97 & 1.00 & 0.81 & 0.86 & 1.00 \\
$B_{\mathrm{H} \alpha \mathrm{p}}$ & 1.880 & $w_{3}$ & 2.31 & 0.97 & 1.00 & 0.81 & 0.85 & 0.99 \\
$B_{\mathrm{H} \alpha \mathrm{p}}$ & 2.115 & $w_{4}$ & 3.20 & 0.97 & 1.00 & 0.81 & 0.85 & 1.00 \\
\hline
\end{tabular}

The remaining rows in Tables 1 and 2 list the results for the cases where the Ho preprocessing was used. A qualitative comparison of the H $\alpha$-preprocessed magnetograms (shown in Figure 5) with the model chromosphere (shown in the top panel of Figure 1) indicates a strong resemblance for all three magnetic field components, but certainly not a perfect match. Difference images between the H $\alpha$-preprocessed magnetograms and the model chromosphere (shown in the top panel of Figure 1) are present in Figure 6. The resemblance using the $\mathrm{H} \alpha$ preprocessing scheme is much improved when compared to the magnetograms resulting from the classical preprocessing scheme.

Table 2 displays metrics of the resulting nonlinear force-free extrapolations using each preprocessing scheme. ${ }^{2}$ As expected, the extrapolation codes perform poorly when the unprocessed boundary $\left(B_{\mathrm{ph}}\right)$ is used. In particular, the resulting magnetic energy $\epsilon_{\mathrm{mag}}$ of this case (normalized to the energy of the reference solution) is only $65 \%$ of the correct answer, making it almost impossible to estimate the free magnetic energy in the solution available for release during eruptive processes such as flares and coronal mass ejections.

Taking preprocessing into account (rows $3-7$ in both tables) significantly improves the result. The force-free consistency criteria $\left(L_{12}, \varepsilon_{\text {force }}\right.$, and $\left.\varepsilon_{\text {torque }}\right)$ are adequately fulfilled for all preprocessed cases and are even better (i.e., have lower values) than the model chromospheric field. This is natural, however, because the preprocessing routine has been developed in particular to derive force-free-consistent boundary conditions from inconsistent (forced, noisy) photospheric measurements. The classical preprocessing $\left(B_{\mathrm{cp}}\right)$ has already reduced the angle to the model $\mathrm{H} \alpha$ fibrils (last two columns of Table 1) by almost a factor of 2, even though no information about the chromosphere has been used. Including chromospheric information (see Figure 4$)$ in our preprocessing routine $\left(B_{\mathrm{H \alpha p}}\right.$, rows $\left.4-7\right)$ significantly reduces the angle of the preprocessed field with the $\mathrm{H} \alpha$ images. The second to last row in Table 1 contains the average angle and in the last column the angle has been weighted by the magnetic field, which means that $\phi_{\mathrm{ave}, w}$ measures mainly how well the magnetic field and the chromospheric fibrils are aligned in regions of high magnetic field strength. For the

\footnotetext{
${ }^{2}$ For an explanation of the extrapolation method used to perform the results in Table 2, see Appendix A and references therein. An explanation of the vector comparison metrics used in the table is given in Appendix B.
} 


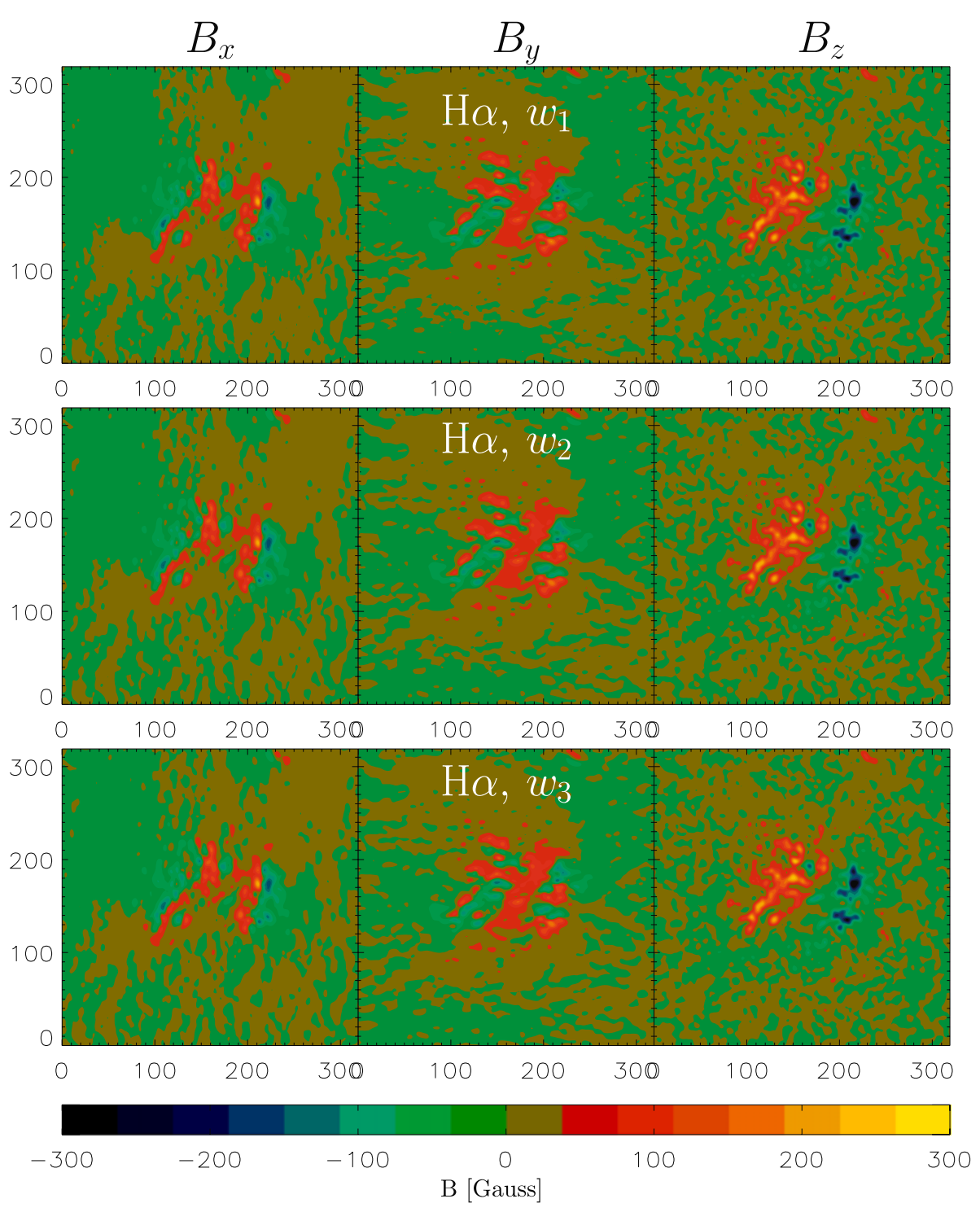

Figure 5 Results of $\mathrm{H} \alpha$ preprocessing with different weighting functions: $w_{1}$ (top), $w_{2}$ (center), and $w_{3}$ (bottom); see text.

purpose of coronal magnetic field extrapolations the strong field regions are essential. If we include all information from the $\mathrm{H} \alpha$ image, as done in row 4 for $w_{1}$, we find that the magnetic field and the fibrils are almost parallel in the entire region. This is the ideal case, however, as fibrils have been identified all over the region with the same excellent accuracy. For observed data it is more likely that the direction of the fibrils will be identifiable with high accuracy only in bright and magnetically strong regions. This effect is taken into account in rows 5-7 of both tables. In the last two rows we take the chromospheric data only into account where the magnetic field strength is larger than $50 \%$ and $10 \%$ of the max- 


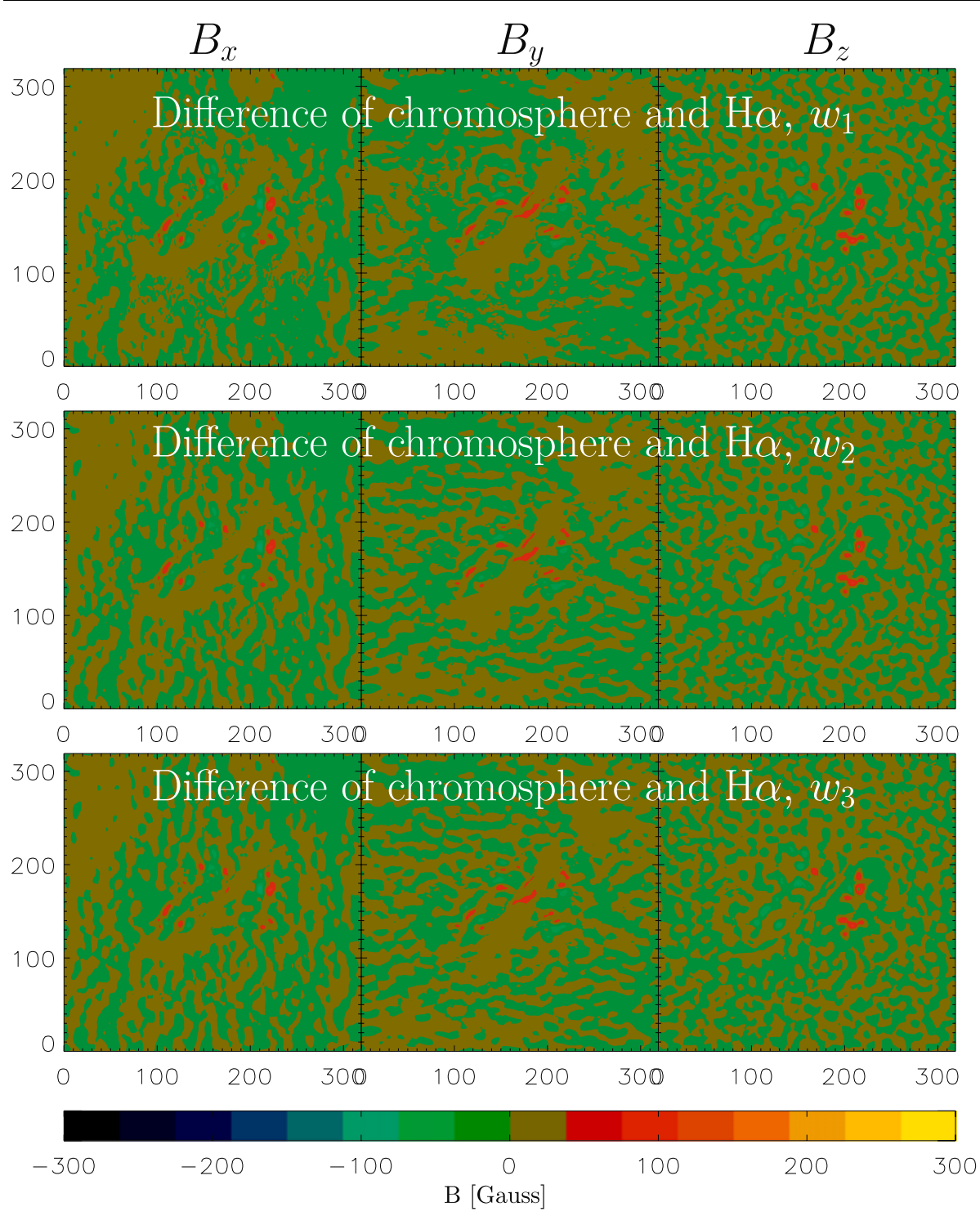

Figure 6 Differences of the chromospheric model field (see top panel of Figure 2 and the Ho-preprocessed fields as shown in Figure 5).

imum field strength, respectively. Naturally, the average angle $\phi_{\text {ave }}$ of the chromospheric fibrils with the preprocessed magnetic field becomes larger than for the ideal case. We find, however, that the angle $\phi_{\mathrm{ave}, w}$ remains relatively low in strong field regions, except for the case $w_{3}$.

We can easily understand that $w_{3}$ (in which chromospheric information is ignored where the magnetic field is less than $50 \%$ of its maximum) provides less accurate results, because the area where chromospheric data have been taken into account is only a very small fraction of the entire region (see Figure 4, lower central panel). 
Case $w_{3}$ has few nonzero points. These points are, however, in the regions with the strongest magnetic field strength. The $L_{5}$ terms minimizes the angle between magnetic field and chromospheric fibrils only in these nonzero points. This local correction does, however, influence the magnetogram globally, because the $L_{1}$ and $L_{2}$ terms contain global measures and the $L_{4}$ terms couples neighboring points. As a consequence the preprocessing result is different from classical preprocessing, even if the $L_{5}$ term is nonzero only for a limited number of pixels.

For observational data the weighting $w_{4}$ (last row in the tables, with areas with less than $10 \%$ ignored; see also lower right panel of Figure 4) seems to be more realistic. In this case the overall average angle is not better than for classical preprocessing but is different by only about $3^{\circ}$ when preferential weighting is given to the more important strong-field regions.

The ultimate test regarding the success of our extended preprocessing scheme is to use the preprocessed field as boundary conditions for a nonlinear force-free coronal magnetic field extrapolation. The results are presented in Table 2 for classical preprocessing (row 3 ) and for $\mathrm{H} \alpha$ preprocessing (rows $4-7$ ). We find that all preprocessed fields provide much better results than those obtained by using the unprocessed data. For classical preprocessing we get the magnetic energy $\epsilon_{\text {mag }}$ correct with an error of $3 \%$ (whereas for unprocessed data we get an error of 35\%). Taking the $\mathrm{H} \alpha$ information into account improves the result and the magnetic energy is computed with an accuracy of $1 \%$ or better, even for the cases where we used chromospheric information only in parts of the entire regions.

\section{Conclusions and Outlook}

Within this work we developed an improved algorithm for the preprocessing of photospheric vector magnetograms for the purpose of getting suitable boundary conditions for nonlinear force-free extrapolations. We extended the preprocessing routine developed by Wiegelmann, Inhester, and Sakurai (2006), which is referred to here as "classical preprocessing". The main motivation for this work is related to the fact that active-region coronal magnetic fields are force-free because of the low- $\beta$ coronal plasma, but the magnetic field vector can be measured with high accuracy only on the photosphere, where the plasma $\beta$ is about unity and nonmagnetic forces cannot be ignored. Our original ("classical") preprocessing removes these nonmagnetic forces and makes the field compatible with the force-free assumption, leading to more chromospheric-like configurations. In this study, we have found that by taking direct chromospheric observations into account (such as by using fibrils seen in $\mathrm{H} \alpha$ images), the preprocessing is improved beyond the classical scheme. This improved scheme includes a term that minimizes the angle between the preprocessed magnetic field and the fibrils. We tested our method with the help of a model active region developed by van Ballegooijen et al. (2007), which includes the forced photospheric and force-free chromospheric and coronal layers. This model has been used by Metcalf et al. (2007) for an intercomparison of nonlinear force-free extrapolation codes. The comparison revealed that the model coronal magnetic field was reconstructed very well if chromospheric magnetic fields have been used as input, but in contrast the reconstructed fields compared poorly when unprocessed model photospheric data were used. Classical preprocessing significantly improves the result, but the $\mathrm{H} \alpha$ preprocessing developed in this paper is even better as the main features of the model corona are reconstructed with high accuracy. Our extended preprocessing tool provides a fair estimate of the chromospheric magnetic field, which is used as boundary condition for computing the nonlinear force-free coronal magnetic field. In particular, the magnetic energy in the force-free domain above the chromosphere agrees with the model 
corona within $1 \%$, even if only strong-field regions of the model chromosphere, where the fibrils can be identified with highest accuracy, influence the final solution. From these tests we conclude that our improved preprocessing routine is a useful tool for providing suitable boundary conditions for the computation of coronal magnetic fields from measured photospheric vector magnetograms as provided for example from Hinode. The combination of preprocessing and nonlinear force-free field extrapolations seems likely to provide accurate computation of the magnetic field in the corona.

We will still not get the magnetic field structure in the relatively thin layer between the photosphere and the chromosphere correct, because here nonmagnetic forces cannot be neglected because of the finite $\beta$ plasma. Although this layer is vertically thin (e.g., 2 vertical grid points in the van Ballegooijen et al. (2007), model compared to 256 vertical grid points in the corona) it contains a significant part of the total magnetic energy of the entire domain; see Metcalf et al. (2007). Unfortunately, this part of the energy cannot be recovered by force-free extrapolations, because the region is non-force-free. Our improved preprocessing routine includes chromospheric information and therefore provides us with a closer approximation of the chromospheric magnetic field. This leads to more accurate estimates of the total magnetic energy in the corona.

A further improvement of the preprocessing routine could be done with the help of additional observations (e.g., the line-of-sight chromospheric field, as planned for SOLIS). One could include these measurement directly in the $L_{3}$ term (14) either as the only information or in some weighted combination with the photospheric field measurement. An investigation of the true 3D structure of the thin non-force-free layer between photosphere and chromosphere requires further research. First steps toward non-force-free magnetohydrostatic extrapolation codes (Wiegelmann and Neukirch, 2006) might help to reveal the secrets of this layer. Non-force-free magnetic field extrapolations will require additional observational constraints, because the magnetic field and the plasma density and pressure must be computed self-consistently in one model.

Acknowledgements The work of T. Wiegelmann was supported by DLR Grant No. 50 OC 0501 and J.K. Thalmann got financial support by DFG Grant No. WI 3211/1-1. M. DeRosa, T. Metcalf, and C. Schrijver were supported by Lockheed Martin Independent Research funds. We acknowledge stimulating discussions during the fourth NLFFF consortium meeting in June 2007 in Paris.

\section{Appendix A: Extrapolation of Nonlinear Force-free Coronal Magnetic Fields}

We briefly summarize our nonlinear force-free extrapolation code here, which has been used to compute the 3D magnetic fields. We solve the force-free equations (1) and (2) by optimizing (minimizing) the following functional:

$$
L=\int_{V}\left[w_{a} B^{-2}|(\nabla \times \mathbf{B}) \times \mathbf{B}|^{2}+w_{b}|\nabla \cdot \mathbf{B}|^{2}\right] \mathrm{d}^{3} x,
$$

where $w_{a}(x, y, z)$ and $w_{b}(x, y, z)$ are weighting functions. It is obvious that (for $w_{a}, w_{b}>0$ ) the force-free equations (1) and (2) are fulfilled when $L$ is zero. The optimization method was proposed by Wheatland, Sturrock, and Roumeliotis (2000) and further developed in Wiegelmann and Neukirch (2003). Here we use the implementation of Wiegelmann (2004), which has been applied to data in Wiegelmann et al. (2005). In this article, we used a recent update of our code that included a multiscale approach 
(see Metcalf et al. (2007) for details). This version of the optimization code (as in this paper) was also used with the model chromospheric, photospheric, and classical preprocessed photospheric magnetic field vector as part of an intercode comparison in Metcalf et al. (2007). For alternative methods to solve the force-free equations (1) and (2) see the review papers by Sakurai (1989), Aly (1989), Amari et al. (1997), McClymont, Jiao, and Mikic (1997), and Wiegelmann (2008) and references therein.

\section{Appendix B: Metrics to Compare a 3D Coronal Magnetic Field with a Reference Solution}

To quantify the degree of agreement between the extrapolated vector fields of the input model field (B, i.e., the extrapolated chromospheric - reference - field) and the nonlinear force-free solutions (b, i.e., the extrapolated preprocessed photospheric field) that are specified on identical sets of grid points, we use five metrics in Table 2 that compare either local characteristics or the global energy content in addition to the force and divergence integrals. These measures have been developed in Schrijver et al. (2006) and subsequently have been used to evaluate the quality of force-free and non-force-free extrapolation codes (Amari, Boulmezaoud, and Aly, 2006; Wiegelmann et al., 2006; Wiegelmann and Neukirch, 2006; Song et al., 2006; Wiegelmann, 2007; Metcalf et al., 2007).

The vector correlation metric has been defined as

$$
C_{\mathrm{vec}}=\frac{\sum_{i} \mathbf{B}_{i} \mathbf{b}_{i}}{\sqrt{\sum_{i}\left|\mathbf{B}_{i}\right|^{2} \sum_{i}\left|\mathbf{b}_{i}\right|^{2}}},
$$

where $\mathbf{B}_{i}$ and $\mathbf{b}_{i}$ are the vectors at each point $i$. One finds that $C_{\text {vec }}=1$ if the vector fields are identical and $C_{\mathrm{vec}}=0$ if $\mathbf{B}_{i} \perp \mathbf{b}_{i}$.

The Cauchy - Schwarz metric is based on the homonymous inequality $(|\mathbf{a} \cdot \mathbf{c}| \leq|\mathbf{a}||\mathbf{c}|$ for any two vectors $\mathbf{a}$ and $\mathbf{c}$ )

$$
C_{\mathrm{cs}}=\frac{1}{M} \sum_{i} \frac{\mathbf{B}_{i} \cdot \mathbf{b}_{i}}{\left|\mathbf{B}_{i}\right|\left|\mathbf{b}_{i}\right|} \equiv \frac{1}{M} \sum_{i} \cos \theta_{i},
$$

where $M$ is the total number of vectors in the volume and $\theta_{i}$ is the angle between $\mathbf{B}$ and $\mathbf{b}$ at point $i$. It is entirely a measure of the angular differences of the vector fields; that is, $C_{\mathrm{cs}}=1$ if $\mathbf{B} \| \mathbf{b}, C_{\mathrm{vec}}=-1$ if they are antiparallel, and $C_{\mathrm{vec}}=0$ if $\mathbf{B}_{i} \perp \mathbf{b}_{i}$ at each point.

The normalized vector error is defined as

$$
E_{\mathrm{n}}=\frac{\sum_{i}\left|\mathbf{b}_{i}-\mathbf{B}_{i}\right|}{\sum_{i}\left|\mathbf{B}_{i}\right|}
$$

The mean vector error averages over relative differences and is given by

$$
E_{\mathrm{m}}=\frac{1}{M} \sum_{i} \frac{\left|\mathbf{b}_{i}-\mathbf{B}_{i}\right|}{\left|\mathbf{B}_{i}\right|} .
$$

Unlike the first two metrics, perfect agreement of the two vector fields results in $E_{\mathrm{m}}=$ $E_{\mathrm{n}}=0$. For an easier comparison with the others, we list $E_{\mathrm{m}, \mathrm{n}}^{\prime} \equiv 1-E_{\mathrm{m}, \mathrm{n}}$, so that all measures reach unity for a perfect match. 
To estimate how well the models rate the energy content of the field, we use the total magnetic energy of $\mathbf{b}$, normalized to the total magnetic energy of $\mathbf{B}$, namely,

$$
\epsilon_{\mathrm{mag}}=\frac{\sum_{i}\left|\mathbf{b}_{i}\right|^{2}}{\sum_{i}\left|\mathbf{B}_{i}\right|^{2}} .
$$

\section{References}

Aly, J.J.: 1989, Solar Phys. 120, 19.

Amari, T., Boulmezaoud, T.Z., Aly, J.J.: 2006, Astron. Astrophys. 446, 691.

Amari, T., Aly, J.J., Luciani, J.F., Boulmezaoud, T.Z., Mikic, Z.: 1997, Solar Phys. 174, 129.

Gary, G.A.: 2001, Solar Phys. 203, 71.

Inhester, B., Feng, L., Wiegelmann, T.: 2007, Solar Phys. in press.

Kramar, M., Inhester, B., Solanki, S.K.: 2006, Astron. Astrophys. 456, 665.

Lin, H., Kuhn, J.R., Coulter, R.: 2004, Astrophys. J. 613, L177.

Low, B.C., Lou, Y.Q.: 1990, Astrophys. J. 352, 343.

McClymont, A.N., Jiao, L., Mikic, Z.: 1997, Solar Phys. 174, 191.

Metcalf, T.R., Jiao, L., McClymont, A.N., Canfield, R.C., Uitenbroek, H.: 1995, Astrophys. J. 439, 474.

Metcalf, T.R., DeRosa, M.L., Schrijver, C.J., Barnes, G., VanBallegooijen, A., Wiegelmann, T., Wheatland, M.S., Valori, G., McTiernan, J.M.: 2007, Solar Phys. accepted.

Molodensky, M.M.: 1969, Sov. Astron. - AJ 12, 585.

Molodensky, M.M.: 1974, Solar Phys. 39, 393.

Sakurai, T.: 1981, Solar Phys. 69, 343.

Sakurai, T.: 1989, Space Sci. Rev. 51, 11.

Schrijver, C.J., van Ballegooijen, A.A.: 2005, Astrophys. J. 630, 552.

Schrijver, C.J., Derosa, M.L., Metcalf, T.R., Liu, Y., McTiernan, J., Régnier, S., Valori, G., Wheatland, M.S., Wiegelmann, T.: 2006, Solar Phys. 235, 161.

Solanki, S.K., Lagg, A., Woch, J., Krupp, N., Collados, M.: 2003, Nature 425, 692.

Song, M.T., Fang, C., Tang, Y.H., Wu, S.T., Zhang, Y.A.: 2006, Astrophys. J. 649, 1084.

van Ballegooijen, A.A.: 2004, Astrophys. J. 612, 519.

van Ballegooijen, A.A., Deluca, E.E., Squires, K., Mackay, D.H.: 2007, J. Atmos. Terr. Phys. 69, 24.

Wheatland, M.S., Sturrock, P.A., Roumeliotis, G.: 2000, Astrophys. J. 540, 1150.

Wiegelmann, T.: 2004, Solar Phys. 219, 87.

Wiegelmann, T.: 2007, Solar Phys. 240, 227.

Wiegelmann, T.: 2008, J. Geophys. Res. 113, A03S02.

Wiegelmann, T., Neukirch, T.: 2003, Nonlinear Process. Geophys. 10, 313.

Wiegelmann, T., Neukirch, T.: 2006, Astron. Astrophys. 457, 1053.

Wiegelmann, T., Inhester, B., Sakurai, T.: 2006, Solar Phys. 233, 215.

Wiegelmann, T., Inhester, B., Lagg, A., Solanki, S.K.: 2005, Solar Phys. 228, 67.

Wiegelmann, T., Inhester, B., Kliem, B., Valori, G., Neukirch, T.: 2006, Astron. Astrophys. 453, 737. 